\title{
Tensiones de género(s) y desafios (trans)feministas: Políticas culturales y gestión pública
}

Gender tensions and (trans)feminist challenges:

Cultural policies and public management

\section{Marcela País-Andrade}

Universidad de Buenos Aires, Buenos Aires, Argentina

mapaisandradedsociales.uba.ar

https://orcid.org/0000-0003-1164-5691

\section{Resumen}

Este artículo explora los disensos, resistencias y negociaciones puestos en juego en lo político del proceso cultural. Concretamente, examina los problemas que se configuran en el campo de las políticas culturales y su gestión pública vinculadas a las cuestiones de género(s)/(trans)feminismos. Aplicando una metodología socioantropológica, se resignifican como desafíos dos ejes problemáticos que emergieron recurrentemente en el proceso investigativo y que han permitido sistematizar y organizar criterios para construir indicadores. Los resultados reflejan cómo el posicionamiento político de visibilizar dichas tramas en los diseños y evaluaciones de las acciones culturales podrían generar información para mejorar los niveles de coordinación y coherencia de dichas acciones. Asimismo, delinean pistas para que el sector identifique los problemas/desafíos relacionados con dichas cuestiones presentes en el campo cultural nacional y/o latinoamericano.

Palabras clave: Cultura, Política Pública, Gestión cultural, Sexualidades, Feminismos.

\begin{abstract}
This article explores disagreements, resistances, and bargaining within the political dimension of cultural process. It particularly examines problems coming out from the field of cultural policies and its public management regarding gender and (trans) feminists' issues. Following a socioanthropological methodological framework, we acknowledge two critical dimensions as challenges, but that they resulted from the fieldwork. The article proposes a better planning and sharper criteria in order to build up a more refined set of metrics. Results show how the political standingpoint of making such plots visible within both designing and assessment in cultural policies could provide better data in order to improve planning and consistency in such policies. Furthermore, cultural players might draw some paths out of our results in order to identify the main challenges related to such issues in the cultural field, both at a domestic and an international level.
\end{abstract}

Keywords: Culture, Public Policy, Cultural Management, Sexualities, Feminisms. 


\section{Introducción}

Las investigaciones de las cuales se desprende este artículo ${ }^{1}$ surgieron en el año 2009. ${ }^{2}$ Se propusieron explorar desde una perspectiva socioantropológica el campo de las políticas culturales y su gestión pública en Argentina, así como también en algunos países de la región. ${ }^{3}$ Dichas pesquisas comenzaron a ser interpeladas, desde el año 2013, por nuestra militancia feminista deviniendo en reflexiones orientadas a observar las cuestiones de género en el campo cultural. ${ }^{4}$ Actualmente, el proceso investigativo se propone fortalecer el estudio de la política cultural y su gestión pública en vínculo con dichas cuestiones, incorporando las diversas disputas sexogenéricas que observan lo político de la sexualidad en el campo cultural/artístico a través de la categoría género(s)/(trans)feminismos. ${ }^{5}$

En este artículo reconstruimos, desde una mirada retrospectiva de nuestro proceso investigativo, ciertas tensiones y dificultades con las que nos encontramos de forma permanente e imbricada a la hora de analizar/intervenir en los procesos de diseño y evaluación de las políticas culturales argentinas. En este sentido, observamos los problemas que se configuran en dicho campo y sus gestiones públicas en vínculo con las cuestiones de género(s)/ (trans)feministas para proponer criterios que permitan mejorar el diseño y la evaluación de las acciones, proyectos y planes culturales actuales. Entendemos que esta visibilización y sistematización brindará pistas para que otrxs colegas identifiquen novedosos desafíos en el campo cultural nacional y/o latinoamericano.

El texto se organiza de la siguiente manera: en primer lugar, se desarrolla el marco teórico y el marco metodológico desde los cuales se ha realizado el recorrido investigativo. Luego, se analizan críticamente los resultados de dichos procesos. En este punto, se resignifican como desafíos dos problemas que han surgido sistemáticamente en el trabajo: al la tensión que se provoca al querer operativizar la política pública en el campo cultural y la elasticidad al querer definir la cultura ly su diversidad) en el campo de la política pública, y b) los disensos que se observan entre las complejidades del campo cultural y lo que efectivamente sucede al interior del sector. Identificar estos ejes ha permitido sistematizar y organizar criterios para que quienes investigan/intervienen en el campo de lo cultural puedan construir indicadores que permitan diseñar y evaluar las políticas culturales y su gestión incorporando las cuestiones de género(s)/ (trans)feminista la mejorar por supuesto). Finalmente, señalaremos algunas conclusiones del trabajo las cuales surgen de nuestra experiencia local (Argentina) pero entendemos que pueden ser representativas y pertinentes para otros países de la región. ${ }^{6}$

\section{Marco teórico}

Concebimos la investigación sociocultural como "el esfuerzo por relacionar distintas dimensiones de un problema analizando los procesos que se generan en sus interdependencias y relaciones históricas contextuales" (Achilli, 2005, p.17). Desde dicho marco, nuestro abordaje se basa en una perspectiva socioantropológica interpelada por las cuestiones de género(s)/(trans)feminista. Dicho diálogo permite observar las disposiciones que suelen configurarse ante los problemas de las personas reales y actores sociales en momentos y espacios específicos. Así, resignificamos dichas destrezas como prácticas de un saber situado y construido desde las tensiones, negociaciones y resistencias de las categorías académicas previas y el saber local (País Andrade \& Igarzábal, 2021).

En este sentido, comprender a las políticas públicas desde la noción de poblaciones-problema (Foucault, 1978) nos acerca al estudio del poder tensionando dicho binomio en tanto términos necesariamente implicados y en conflicto (Shore \& Wright, 1997; Shore, 2010). Además, en las tramas en las que se insertan nuestras investigaciones - donde se problematizan los vínculos entre lo cultural, lo político, lo público, el poder, las identidades y las sexualidades - requerimos problematizar y deconstruir de manera crítica los procesos de identidad y diversidad cultural (Wortman, 1996; Lacarrieu, 2008; Lins Ribeiro, 2009; Calabre, 2009; Rebello, 2010; Barbieri, Partal \& Merino, 2011; Mariscal, 2012) que se van reconfigurando en relación a las cuestiones de género (Di Marco, 1997; Bonder, 1998) en el marco de los diseños, gestiones y/o evaluaciones junto a las maneras en que se desarrollan los programas, proyectos y líneas de acción culturales en vínculos con las inequidades socio/ étnico/genéricas (Falquet, 2003; Gargallo, 2014; Segato, 2015). 
Al dialogar estos enfoques, se observó la necesidad de dar cuenta de las distintas formas de politización de la cultura (Wright, 2004). Las mismas van reproduciendo cuestiones políticas - sea explícito o noal mismo tiempo que van reconfigurando conocimiento histórico, social, localizado y humanizado; es decir, una teoría de la existencia social misma (Quijano, 2007). En esta conversación, propusimos entender las formas de la vida misma y cómo nosotrxs nos vinculamos con ella. Es decir, identificar las maneras en que esas relaciones son construcciones contingentes que están complejamente ligadas con la organización de la cultura y el poder.

Como resultado de estos debates comprendimos que en el diseño y ejecución de la política cultural pública se cristaliza el conjunto de estrategias organizadas y jerarquizadas por el Estado, que nos permiten entender analíticamente el sentido que se le da a la noción de cultura (y su gestión) en una coyuntura política y social específica. Esto, se pone en juego en dos niveles: por un lado, se operativiza en y desde los diversos actores que detentan el poder estatal linstituciones, organismos, personas que ejercen funciones públicas, mercado, grupos sociales, etcéteral dándole sentido a lo que se entiende por lo cultural en un proyecto político dado. Paralelamente, se materializa en las prácticas disruptivas de y desde lxs diversxs actores que visibilizan la exclusión e inequidad que se configura en dicha proyección cultural. De esta forma, el campo cultural se encuentra en un permanente proceso destinado a reforzar, rechazar, confrontar y/o negociar estrategias identitarias que les dan sentido político a las prácticas cotidianas (País Andrade, 2016).

Consecuentemente, nuestro sentido político de las prácticas culturales nos obligó a reflexionar sobre la diversidad/desigualdad cultural (Reygadas, 2007; Fraser, 2009) y, específicamente, a complejizar dichos estudios desde las cuestiones de género(s) y su imbricación con las políticas públicas. Por tanto, sumamos a este corpus los trabajos feministas y los de raza para pensar desde enfoques interseccionales (Crenshaw, 1989; Hall, 1992; Viveros, 2016). Como resultado, hemos construido la categoría de género(s)/(trans)feminismos para dar cuenta de las implicancias políticas del uso cultural de ciertos términos sociosexuales. En consecuencia, referimos a género(s) para desbordar el binomio femenino-masculino e incorporar las identidades trans que implican la materialización de diversas formas de ser y sentirse mujeres o varones. Asimismo, resignificamos la idea de (trans)feminismos desde el enfoque transfeminista de Sayak Valencia (2018), quien lo entiende como "una red que considera los estados de tránsito de género, de migración, de mestizaje, de vulnerabilidad, de raza y de clase, para articularlos como herederos de la memoria histórica de los movimientos sociales de insurrección." (p.31). Ambas nociones nos permiten configurar la categoría de género(s)/(trans)feminismos para construir un aporte teórico-metodológico que permita visibilizar y poner en diálogo las distintas genealogías políticas y teóricas de cada uno de estos campos. Si bien no es intención de este artículo profundizar en estos debates subrayamos que -en Latinoamérica- los trabajos de género, los estudios trans y el movimiento feminista, no necesariamente han desarrollado los mismos caminos y criterios abriéndose entre sí ly al interior de cada campol críticas, pugnas y distancias en relación a las formas de entender, negociar, negar, discernir y/o desafiar a la política pública y al Estado.

Resumiendo, en este artículo exploramos las complejidades empíricas que han surgido en la dialéctica entre las discusiones teóricas-metodológicas y el trabajo de campo; resignificándolas como desafíos a tener en cuenta para construir criterios que permitan mejorar el diseño y la evaluación de las acciones, proyectos y planes culturales. A los fines analíticos los hemos dividido en dos, a saber: al la tensión que se provoca al querer operativizar la política pública en el campo cultural y la elasticidad al querer definir la cultura (y su diversidad) en el campo de la política pública ( en esta compleja relación dialéctica se observa (sobre todo) al momento de diseñar y/o ejecutar las acciones culturales);y b) Los disensos que se observan entre las complejidades del campo cultural y lo que efectivamente sucede al interior del sector.

\section{Marco Metodológico}

La metodología socioantropológica de los estudios realizados se basan en una mirada relacional y multisituada en tanto focalizamos en las relaciones sociales implicadas en las dinámicas locales (Shore, 2010). Dicha metodología parte del supuesto de que todo proceso social humano se desarrolla inscrito en relaciones sociales, que pueden ser de 
diverso carácter: simétricas, asimétricas, conflictivas, no conflictivas, etcétera. Por tanto, nuestra experiencia de trabajo, basada fuertemente en la etnografía -entendida como la presencia directa y sostenida del investigador en el lugar donde se encuentran los actores/miembros de la unidad sociocultural que desea estudiar (Guber, 1991) - fue una instancia central para la producción de conocimiento: la participación como formadora y capacitadora -desde el año 2013 hasta la actualidad-en el Programa de Formación en Gestión Cultural Pública (PFGCP) dependiente de la Dirección Nacional de Innovación Cultural del Ministerio de Cultura de la Nación y las estancias de investigación sostenidas en diferentes regiones del país, me permitieron "estar ahí" en el diseño, ejecución y evaluación de acciones que se desarrollaron a lo largo y ancho del territorio. Específicamente para este artículo profundizamos en experiencias territoriales de formación, capacitación e investigación realizadas en las provincias del Noroeste (NOA) y Noreste (NEA) de Argentina, pues son las regiones donde más tiempo hemos sostenido el trabajo en campo.

Nuestro corpus está conformado por la revisión de fuentes secundarias desde el 2013: al sistematización de las actividades del PFGCP; relevamiento de los espacios y actividades culturales que se han recuperado desde el sistema de Información Cultural de la Argentina (SINCA), específicamente de las regiones mencionadas; folletos de las actividades, material periodístico, audiovisual y en redes sociales acerca de las actividades que se realizaron durante períodos concretos, entre otros; $b$ ) información cuantitativa que brinda la Segunda Encuesta de Consumo Cultural a nivel nacional (la primera fue en 2013). Esta, por primera vez, elabora un informe específico sobre la condición de las mujeres en el campo cultural al que denominó: “Mujeres en la cultura. Notas para el análisis del acceso y la participación cultural en el consumo y el mercado de trabajo". ${ }^{7} \mathrm{Y}$ cl revisamos leyes vinculadas a las distintas acciones del Estado en cuanto a sexualidades. ${ }^{8}$

Además, las técnicas utilizadas en la investigación fueron: al entrevistas en profundidad a diferentes funcionarixs, artistas y gestorxs culturales de las regiones mencionadas, conformando un total de 15 fuentes clave; b) 10 observaciones participantes y no participantes en organismos culturales con el fin de ser parte de los momentos organizativos y/o decisorios de lxs diseños, ejecuciones y evaluaciones de proyectos culturales, como también de registrar diálogos, actitudes, prácticas, gestos de las distintas personas en otros espacios como eventos culturales, conversatorios, talleres y cursos; c) más de 40 charlas formales e informales con personas clave del sector cultural (referentes de las actividades culturales nombradas y referentes de la gestión de políticas culturales) para identificar ejes problemáticos en el campo vinculados a las cuestiones sociogenéricas. Las mismas han sido producto de un muestreo no probabilístico -entre el 2013 y mediados de 2020 - conocido como bola de nieve en la que la autora de este escrito - como formadora en el PFGCP, participante de la Red de Gestión Cultural Argentina e investigadora del campo cultural- comenzó a contactar a diversxs artistas/gestorxs para el estudio quienes, a su vez, fueron incorporando artistxs/gestorxs nuevxs que conocían y éstxs, a otrxs; d) Dichas experiencias han sido analizadas cualitativamente con el propósito de observar las diversas estrategias que desarrollaron los grupos y/o artistas/gestorxs de forma individual a fin de transversalizar las demandas (trans)feministas de la época en el sector cultural en el cual se desarrollan. Las unidades de análisis seleccionadas congregaron a artistas, gestorxs culturales, funcionarixs, empleadxs y otrxs agentes de las instituciones -oficiales y no oficiales; de nivel nacional, provincial, municipal- involucradxs en la implementación de prácticas, programas, proyectos y líneas de acción que recuperaran las perspectivas centradas en el reconocimiento de las diversidades (sexuales, étnico/raciales, etarias, otras).

\section{Resultados de los estudios em- píricos: resignificando las dificul- tades como desafíos posibles}

El 10 de diciembre del año 2015 asumió la presidencia de la Nación Argentina Mauricio Macri representando a la Coalición política "Cambiemos". La finalizada gestión (10 de diciembre de 2019) implementó acciones políticas, económicas y socioculturales enmarcadas en discursos con tintes liberales, desarrollistas y conservadores. Los años del "Macrismo" (2015-2019) reconfiguraron económica y socialmente los sentidos de lo político, interpelando los programas, proyectos y/o líneas de acción que se venían produciendo durante el período Kirchnerista (2003-2015).9 Dicha trama, se resignificó en el 
campo cultural en y desde la noción del emprendedurismo (Comaroff \& Comaroff, 2011), reivindicando y profundizando ciertas políticas de empoderamiento - proyectadas por los Organismos Internacionales lla Organización de Naciones Unidas; las Naciones Unidas para la Educación, la Ciencia y la Cultura; el Banco Interamericano de Desarrollo, entre otros)que fueron profundizadas y desplegadas sin sentido crítico.

Pese al escenario descrito, en esos años se fortalecieron los movimientos de la sociedad civil para resistir y defender los derechos adquiridos durante los gobiernos anteriores (2003-2015). En este sentido, los grupos que subsistieron a la reorganización y/o desfinanciamiento de programas y directrices públicas (diversidades sexuales, migrantes, etnias, entre otros) resultaron estratégicos como dispositivos de resistencia, de lucha y de transformación de la realidad social. Sin embargo, dichas acciones gubernamentales a nivel nacional lograron permear de formas particulares (falta de presupuesto, vaciamiento de tareas, disminución de recursos humanos, etc.) los vínculos entre el diseño, la ejecución y la evaluación de las acciones culturales (Vich, 2014; Yáñez, 2014; País Andrade, 2016; Peters, 2019; Fuentes \& Tasat, 2019; Molina, 2021) y las cuestiones de género(s)/(trans)feminismos. Sobre todo, ha llamado la atención cómo los movimientos de \#NiUnaMenos desde el año 2015 y la "Ola Verde" feminista del 2018 generaron una fuerte visibilización de lo político de la sexualidad (Rubin, 1989), en el ámbito de las políticas culturales y sus gestiones.

\subsection{Campos problemáticos}

Teniendo en cuenta el contexto sociopolítico argentino observado, en los apartados siguientes reconstruiremos algunas limitaciones y posibilidades al momento de pensar en el diseño y la evaluación de las acciones culturales públicas observadas en clave de género(s)/(trans)feminismos. Para ello, hemos reconstruido analíticamente dos grandes campos problemáticos identificados en nuestros trabajos a partir de situaciones, narraciones y preocupaciones que se repetían en las entrevistas, observaciones en campo y el "estar ahí" de nuestras investigaciones. La intención es resignificarlos como desafíos actuales del sector para construir criterios empíricos que permitan mejorar el diseño y la evaluación de acciones, proyectos y/o planes culturales (y sus gestiones) imbricando las complejidades cotidianas y políticas que implican las cuestiones sexogenéricas. Dichos criterios los organizamos como preguntas metodológicas y teóricas -en un cuadro que cierra este apartado- para ser puestas en diálogo con las prácticas de intervención e investigación de quienes gestionan lo cultural en la actualidad.

\subsubsection{Lo Político del diseño y la ejecución de las acciones culturales públicas}

El primer eje problemático que identificamos en este artículo se convirtió en uno de los principales problemas en nuestro trabajo de campo al interrogar(nos) sobre: ¿Qué entienden lxs diferentes actores del campo cultural por política cultural pública? ¿Quienes participan? ¿Quiénes quedan afuera? y, más específicamente, ¿qué entienden por "cultura"? Algunos ejemplos que podemos citar -en las voces de las personas que hemos entrevistado- acerca de las razones de las que dan cuenta quienes han gestionado proyectos 0 programas específicos cuando naufragan sus propuestas son: "[...] en ese espacio no les interesa lo propuesto porque no tienen 'cultura' para apreciar el producto ofrecido" (trabajadora cultural, 2016); "[...] hay que acercarles la cultura de alguna otra forma [...], tenemos que ser más creativos" (trabajador cultural, 2016); “[...] fue gratuito y público y de todas maneras no participaron; ya no sabemos cómo hacer para que vengan" (artista, 2016); "desde la Secretaría hicimos un diagnóstico exhaustivo de lo que no había en la ciudad, invertimos recursos financieros y humanos en crear el Museo y no va nadie" (funcionario, 2016). ${ }^{10}$

Por medio de estas breves expresiones ejemplificamos cómo las personas que trabajan en el ámbito de la cultura, muchas veces, tienen la idea de que la cultura es algo dado, estático, ahistórico, homogéneo, universal, sin diferencias sociales y/o sociogenéricas en los diversos grupos humanos. Nos permite observar, además, que en la actualidad y en nuestro territorio nacional, que quienes gestionan lo cultural (País Andrade, 2016) -en generalenmarcan las acciones que realizan cabo desde la combinación de ciertos paradigmas Isabiéndolo o no): el de la cultura como recurso (Yúdice, 2002), el de la democratización cultural (García Canclini, 2005) y/o el de la cultura participativa (Vich, 2018). Estas experiencias, junto a otras, nos permitieron 
acordar - con quienes habitan los espacios culturales (por medio de talleres, charlas, diseños conjuntos de acciones culturales, entrevistas, entre otras) - que la cultura es culturas (Geertz, 1987) y, por tanto, es proceso; que en su definición se imbrican realidades muy diversas; que contiene la necesidad de entablar criterios de convivencia, ciudadanía, tolerancia y respeto (Miller \& Yúdice, 2004), y que, además, se propone como campo de derechos humanos que transforma y/o reproduce, incluye/ excluye, etcétera. Así, la inherente presencia de lo político en el campo cultural (Giménez, 2007) sustenta que el proceso de diseño y de desarrollo de una política cultural sea una decisión política.

De esta manera, lo que se entenderá por política cultural y su gestión pública permitirá identificar a los agentes culturales relevantes con quienes dialogar en función del programa y/o proyecto particular. Éstos deberían contar con procesos diagnósticos críticos que identifiquen previamente a dichos agentes, quienes serán los que facilitarán las redes participativas para la operatividad de la acción decidiendo cómo participarán de los momentos de diseño, gestión y/o evaluación. Estos pueden ser personas que trabajen en la función pública, burocracias intelectuales, personas creadoras, artistas, personas que trabajen en el sector empresarial, públicos y asistentes (Ejea, 2011). A los actores sociales nombrados, debemos sumarle: las políticas públicas y los proyectos nacionales, provinciales y municipales vigentes, los diversos colectivos socioculturales, los movimientos y/o comunidades específicas /como las de derechos humanos, mujeres, varones, no binaries, personas transgénero e intersexuales, indígenas, migrantes, movimientos por la tierra, en la actualidad no podemos dejar de pensar en los movimientos sanitarios y ecológicos que se profundizan en el contexto de pandemias mundiales, entre otros).

\subsubsection{Lo político en la ejecución y evaluación del sector cultural}

Las complejidades anteriores nos obligaron a recuperar las formas en que el campo de la cultura pública argentina ha encontrado para medir/evaluar los distintos procesos e impactos sociogenéricos en las acciones culturales de la última década. Esto nos ha permitido indagar en los disensos que se observan entre las complejidades del campo cultural y lo que efectivamente sucede al interior del sector.
En el año 2013, la Secretaría de Cultura realizó la Primera Encuesta Nacional de Consumos Culturales por medio del Sistema de Información Cultural de la Argentina (SInCA), dependiente de la Dirección Nacional de Industrias Culturales y se transformó en el instrumento privilegiado en brindar información estratégica para tomar decisiones en el campo cultural. En ella se dio cuenta de los gustos, las preferencias, los usos, las percepciones y las valoraciones que tiene la ciudadanía en relación a la cultura, en general, y de los consumos culturales en particular.

En el año 2017, se aplicó la Segunda Encuesta a nivel nacional la cual, por primera vez, considera cuestiones de género y elabora un informe específico sobre la condición de las mujeres en el campo cultural al que denominó: “Mujeres en la cultura. Notas para el análisis del acceso y la participación cultural en el consumo y el mercado de trabajo" (SInCA, 2018). Dicho informe se presenta con el objetivo de convertirse en

\footnotetext{
un avance de la investigación sobre la perspectiva de género en el sector cultural, analizada desde la óptica del acceso, el consumo y la participación cultural, por un lado; y del mercado laboral, el empleo del sector cultural y las profesiones culturales por otro (p. 3).
}

Recomendamos consultar la publicación online del informe donde se pueden observar con claridad los datos a partir de gráficas y tortas porcentuales ya que por cuestiones de espacio editorial no podemos explayarnos- $y$, así, vislumbrar con mayor claridad los datos destacados.

Dos cuestiones por demás importantes se desprenden del trabajo señalado: por un lado, el informe es una oportunidad para visibilizar el lugar de mujeres y hombres tanto en el acceso a la participación del campo cultural como a las condiciones de quienes trabajan en dicho espacio - retoma y visibiliza, por cierto, las incomodidades que veníamos registrando en nuestras experiencias de campo-; por otro, nos deja un sinsabor cuando observamos que los datos no nos permiten dar cuenta de la situación actual de los colectivos vinculados a las diversidades y/o disidencias en el campo de la cultura argentina. Del mismo modo, tampoco observa las relaciones políticas/activistas/militantes que tienen dichos colectivos que desde la sociedad civil resisten/tensionan/ 
demandan reconocimiento político para el acceso al propio campo cultural que disputan llo cual pudimos ir observando con mayor fuerza a partir del 2015). Por otro lado, no da cuenta de las formas en que se reproducen las prácticas sexistas manifiestas en las tensiones, negociaciones y resistencias del trabajo vinculado a lo femenino y/o feminidades en el propio sector. Lo dicho se pudo observar en las gestiones culturales situadas donde sobrevolaban las inquietudes relacionadas con las incomodidades e inseguridades de qué hacer con las demandas de los distintos grupos artísticos feministas/disidentes/ diversos que resignifican temas que hasta ahora no habían sido ponderados por el campo de las políticas culturales públicas y su gestión. Algunos puntos que afloraron en las experiencias de campo y las charlas formales e informales que hemos realizado fueron: cómo incorporar la perspectiva de género a los proyectos culturales $y / 0$ a las propias prácticas de las políticas en sus instituciones; qué hacer con las demandas juveniles y/o de los diferentes grupos que militan cuestiones de género y los feminismos en nuestros territorios de acción; cómo los nombramos; cómo materializamos en demandas la desigualdad al interior del sector relacionadas con las contrataciones e inequidad salarial en tanto mujeres, varones, trans; entre otras.

En este sentido, el informe señalado nos permite observar dónde y cómo estamos. No obstante, falta la pregunta que incluya a las diversidades/disidencias y muestre la situación laboral y/o de acceso en y al campo cultural de todas las personas según su condición sexogenérica y las inequidades presentes.

Asimismo, permite repensar la herramienta evaluativa como un proceso de construcción social de conocimiento en el campo de las políticas y su gestión. Siendo parte constitutiva de un escenario específico y desde el cual se resignifica como una categoría de análisis propia de dichas trayectorias. Por eso necesitamos reflexionar nuestros instrumentos de medición cultural en el propio proceso de indagación de las políticas públicas con las cuales intervenimos/ investigamos implicando que los mismos sean diseñados de manera contextualizada, situada y específica. De esta forma, al preguntarnos, siguiendo a Cris Shore, “ ¿qué quiere decir política pública en este contexto? ¿Qué funciones tiene? ¿Qué intereses promueve? ¿Cuáles son sus efectos sociales? Y ¿cómo este concepto de política pública se relaciona con otros conceptos, normas o instituciones dentro de una sociedad en particular?" (2010, p.29). Debe- mos incorporar a la reflexión, también, las siguientes preguntas: ¿Qué vamos a evaluar en y desde dicha acción? ¿Para qué vamos a evaluar? ¿Quiénes van a evaluar? ¿A quiénes se va a evaluar y por qué?, entre otras.

Sabemos que la evaluación siempre es relativa a los parámetros y/o referencias que nos hemos propuesto lograr (lo cual puede ir modificándose y/o re-direccionarse durante el desarrollo de ciertas acciones para lograr los objetivos previstos). La evaluación requiere también de la creatividad de quienes implementan estas tareas, de las redes construidas, de los recursos y del presupuesto disponible. Generalmente, la evaluación presenta diversas complejidades: ¿Cómo?, ¿cuándo? ¿quiénes?, ¿para qué?, etcétera. Sin embargo, es necesario relevar los procesos evaluativos como herramientas necesarias para monitorear la direccionalidad de las acciones, detectar aciertos/problemas/dificultades durante su desarrollo y el impacto final del mismo. Que se conviertan en herramientas "observadoras" de los propios procesos.

En esta línea, entender la evaluación como comprensión y no como valoración (Barbieri, Partal \& Merino, 2011); reflexionar dicho proceso como técnica y política de trayectorias institucionales; observarla de manera tal que permita: la conceptualización y la formulación de criterios sustantivos que sean eficaces, pero también eficientes para comprender los cambios socioculturales que los territorios y el propio sector demanda; la identificación de las dificultades subjetivas que eso cambios encuentran; la habilidad de pensar indicadores que observen los aspectos de esos territorios; y, también los cambios de época que se demandan. Comprender estas cuestiones nos lleva a recuperar perspectivas multidimensionales, de género(s), (trans)feministas e interseccionales para generar procesos evaluativos sólidos que sean coherentes con metodologías participativas e inclusivas.

\subsection{Criterios para diseñar y evaluar acciones, proyectos y/o planes culturales ly sus ges- tiones) transversalizados por las cuestiones de género(s)/(trans)feminismos}

Siguiendo las ideas fuerza que hemos retomado de nuestros trabajos empíricos en el punto anterior, construimos ciertos criterios que hemos organizado y sintetizado - en y desde la dialéctica entre las 
voces de los territorios andados y las teorías discutidas - en el siguiente cuadro en forma de interrogantes que intuimos podrían ser útiles en la cons- trucción de indicadores perspicaces para diseñar y/o evaluar nuestras acciones culturales gestionando feminismos.

\section{Cuadro 1. Preguntas para transversalizar las cuestiones de género(s)/(trans)feminismos}

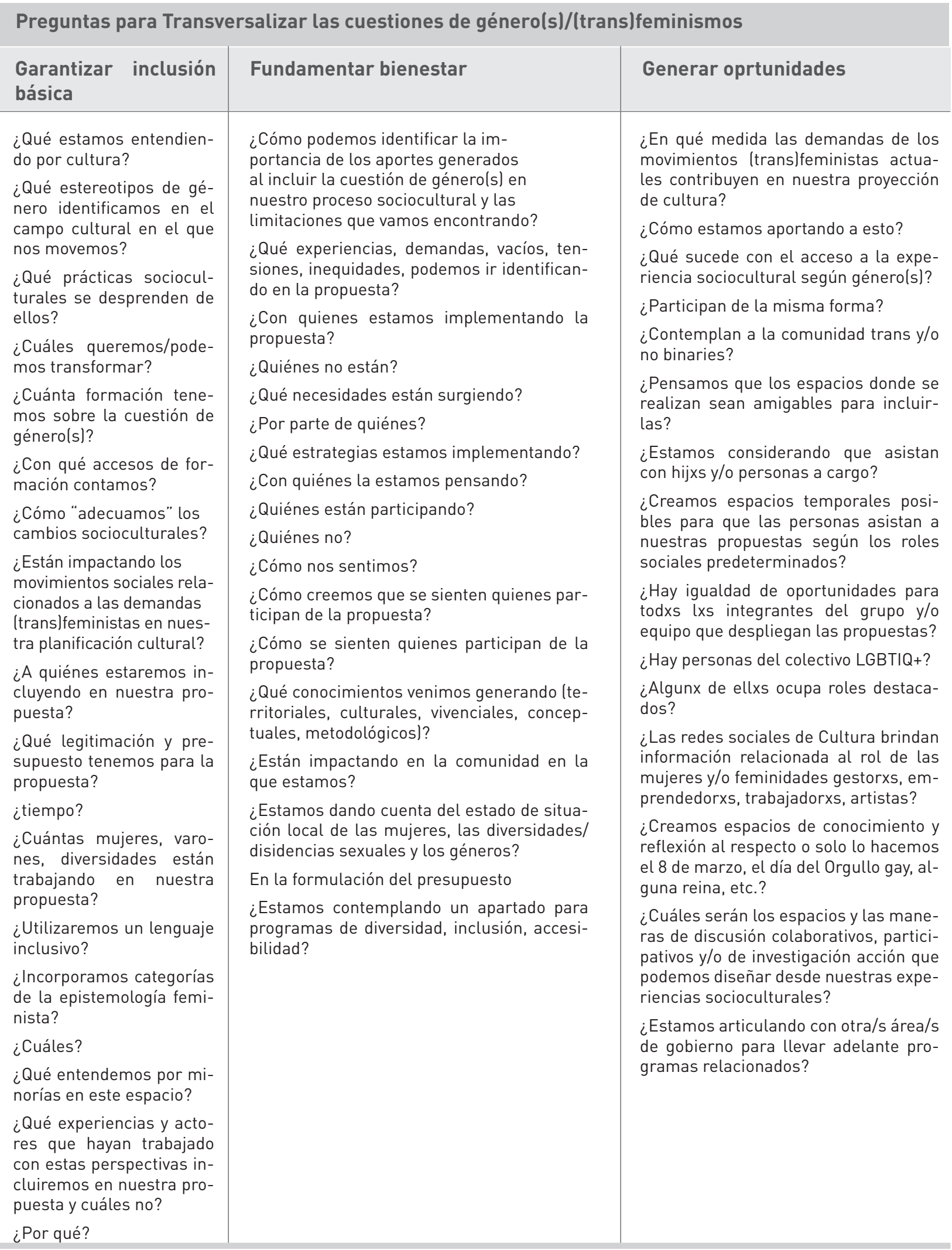

Fuente: Elaboración propia a partir del trabajo de campo desarrollado entre 2016-2019. 


\section{Conclusiones}

Reflexionar sobre los problemas que hemos transitado en nuestro proceso investigativo en el campo de las políticas culturales y sus gestiones imbricando las cuestiones de género(s)/(trans)feminismos - que hemos reconfigurado en términos de desafíos-, nos permitió proponer ciertos criterios a tener en cuenta para mejorar los procesos de acciones, proyectos y planes culturales. Dichos puntos demuestran que el posicionamiento político de visibilizar y tensionar las identidades sexogenéricas en los procesos de diseño y evaluación podrían generar información para mejorar los niveles de coordinación y coherencia entre los diferentes programas, planes y proyectos en el campo cultural argentino (con posibilidades de ser un aporte para toda la región). Intuimos, también, que facilitarían el diálogo con otras políticas socioculturales destinadas a las mismas poblaciones. Entendemos que reflexionar sobre los procesos de diseño y evaluación en esta clave implica, por un lado, la deconstrucción de ciertas categorías tradicionales universalistas - sobre cómo diseñar y evaluar- desde categorías que provienen de la epistemología feminista. Por otro lado, nuestro contexto nacional/regional/mundial exige ponerse en diálogo con metodologías situadas y participativas provenientes de un conocimiento local y comunitario que dé cuenta de las complejidades cotidianas que se configuran en torno a las diversidades culturales y la reivindicación de derechos en el crítico contexto socio-sanitario actual. Este giro contribuirá al campo de las políticas culturales de gestión pública, que sean situadas y pertinentes a las comunidades a las que se proponen servir.

\section{Notas}

1. Agradecemos a la Facultad de Ciencias Sociales de la Universidad de Buenos Aires (UBA) y al Consejo Nacional de Investigaciones Científicas y Técnicas (CONICET) por los reconocimientos y los subsidios otorgados a los diferentes proyectos que son la base fundamental de este artículo.

2. El primer grupo de investigaciones a las que referimos han quedado plasmadas en el libro: Cultura, Ju- ventud, Identidad: una mirada socioantropológica del Programa Cultural en Barrios (País Andrade, 2011).

3. Damos cuenta de estas pesquisas en el libro Cultura y desarrollo en América latina. Actores, estrategias, formación y prácticas (País Andrade y Molina Roldán, 2013).

4. La interpelación de la perspectiva de género(s) al estudio de la Política Cultural y su gestión se observan en el libro Identidades culturales en y desde las fronteras. Un enfoque de género a la(s) políticas y a la(s) prácticals) culturales juveniles (País Andrade, 2016).

5. Se puede profundizar en nuestro reciente trabajo De la cultura al feminismo (País Andrade \& Igarzábal, 2021).

6. Para Joan Scott (1988), la experiencia puede entenderse como el resultado de un complejo entramado de representaciones sociales que circulan en la sociedad y la cultura, la experiencia como producto histórico, social y cultural.

7. Se puede ver el informe completo en este sitio:https://www.sinca.gob.ar/VerDocumento. aspx?IdCategoria=1

8. Ley 25.673 de Creación del Programa de Salud Sexual y Procreación Responsable (2002); ley 26.150 de Educación Sexual Integral (2006); la ley 26.485. Protección Integral para Prevenir, Sancionar y Erradicar la Violencia contra las Mujeres en los Ámbitos en que Desarrollen sus Relaciones Interpersonales (2009); la modificación del artículo 2 de la ley 26.618 de Matrimonio Civil (conocida como la Ley de Matrimonio Igualitario en 2010); la ley 26.743 de Identidad de Género (2012); la ley 26.842 de Prevención y sanción de la trata de personas y asistencia a sus víctimas (2012); y en la actualidad (2020), la ley 27.610 de Interrupción Voluntaria del Embarazo (IVE); la ley 27.611 de Atención y cuidado integral de la salud durante el embarazo y la primera infancia (conocida como la Ley de los Mil Días) y la ley 27.636 de Promoción del acceso al empleo formal para personas travestis, transexuales y transgénero “Diana Sacayán Lohana Berkins" (2021).

9. Es en dicha década donde se comienza a configurar el proceso político de origen peronista denominado Kirchnerismo. En efecto, en este período se delinearon ciertos programas, proyectos y líneas de acción caracterizados, en términos generales, por discursos que rechazaban las miradas del neoliberalismo, las políticas económicas desarrollistas y los tratados del libre comercio. Se promulgaron (con matices por supuesto) políticas públicas destinadas a la defensa del Mercosur, el alineamiento internacional latinoamericano y la revalorización de los Derechos Humanos. En esta última línea, se reivindicaron ciertas acciones y/o programas gubernamentales garantes de derechos a las mi- 
norías vulneradas como grupos migrantes, indígenas, juventudes, mujeres, diversidades sexuales, LGBTTTIQ+, entre otros. ("Autor/a", 2018).

10. Estas afirmaciones han sido registradas y sistematizadas durante el mes de octubre del año 2016 en el Marco del Programa de Formación en Gestión Cultural Pública (Dirección Nacional de Formación Cultural) del Ministerio de Cultura de la Nación. Hemos elegido estas citas por ser representativas de ciertas nociones que se repiten cotidianamente en los distintos ámbitos de formación y espacios de discusión con artistas y/o trabajadorxs de la cultura.

\section{Referencias}

Achilli, E. (2005). Investigar en antropología social. Los desafíos de transmitir un oficio. Laborde Editor.

Barbieri, N., Partal, A., \& Merino, E. (2011). Nuevas políticas, nuevas miradas y metodologías de evaluación. ¿Cómo evaluar el retorno social de las políticas culturales? Papers, (96) 2, 477- 500. http://dx.doi.org/10.5565/rev/papers/v96n2.139

Bonder, G. (1998). “Género y Subjetividad: avatares de una relación no evidente”. En S. Montecino \& A. Obach (Ed.), Género y Epistemología. Mujeres y disciplinas. Universidad de Chile.

Calabre, L. (2009). Políticas Culturais no Brasil: dos anos 1930 ao século XXI. FGV.

Comaroff, J. \& Comaroff, J. (2011) Etnicidad SA. Katz.

Crenshaw, K. (1989). Demarginalizing the Intersection of Race and Sex: A Black Feminist Critique of Antidiscrimination Doctrine, Feminist Theory and Antiracist Politics. University of Chicago Legal Forum, (1)1989, 139-167. https://chicagounbound.uchicago.edu/ uclf/vol1989/iss1/8

Di Marco, G. (1997). “Las mujeres y la política en los '90”. En B. Schmukler \& G. Di Marco (Comps.), Madres y democratización de las familias en la Argentina contemporánea, Biblos.

Ejea, T. (2011). Poder y creación artística en México. Un análisis del Fondo nacional para la Cultura y las Artes (FONCA). Universidad Autónoma Metropolitana Azcapotzalco.

Falquet, J. (2003). Mujeres Feminismo y Desarrollo. Mujeres feminismo: un análisis crítico de las políticas de las instituciones internacionales. Desacatos, (11), 13-35.

Foucault, M. (1978). "La «gouvemementalité»"; curso del College de France, año 1977-1978. «Seguridad, territorio y población», (167-168), 12-29.

Fraser, N. (2009). Feminism, Capitalism and the Cunning of History. New Left Review, (56), $97-$ 117.

Fuentes, E. \& Tasat, J. (Comps.). (2019). Gestión Cultural en Argentina. RGC libros.

García Canclini, N. (2005). “Todos tienen cultura. ¿Quiénes pueden desarrollarla?”. Conferencia para el Seminario sobre Cultura y Desarrollo en el Banco Interamericano de Desarrollo. Washington, Georgetown University.

Gargallo, F. (2014). Feminismos desde Abya Yala. Ideas y Proposiciones de las Mujeres de 607 Pueblos de Nuestra América. Ed. Corte y Confección.

Geertz, C. (1987). La interpretación de las culturas. Gedisa.

Giménez, G. (2007). Estudios sobre la cultura y las identidades sociales. CONACULTA/ITESO. 
Guber, R. (1991). El salvaje metropolitano. Editorial Legasa.

Hall, S. (1992). Sin garantías. Trayectorias y problemáticas en estudios culturales. Envión Editores-IEP- Instituto Pensar-Universidad Andina Simón Bolívar.

Lacarrieu, M. (2008). “Identidad, Diversidad Cultural y Globalización. La gestión de la diversidad cultural y las identidades en el contexto de la globalización. Retos y Desafíos para las políticas públicas de la cultura". Primer Congreso Argentino de Cultura (pp.171187). Secretaría de Cultura de la Nación, Instituto Cultural de la Provincia de Buenos Aires.

Lins Ribeiro, G. (2009). Diversidade cultural enquanto discurso global. Ava, Revista de Antropología, (15), Universidad Nacional de Misiones. https://www.redalyc.org/comocitar. oa?id=169016753001

Mariscal, J. L. (Coord.) (2012). Profesionalización de gestores culturales en Latinoamérica. Estados, Universidades y Asociaciones. UDGvirtual.

Miller, T. \& Yúdice, G. (2004). Política cultural. Gedisa.

Molina, A. (2021). Gestión y estudios culturales: un encuentro posible, un desafío permanente. Antrópica. Revista de Ciencias Sociales y Humanidades. 7 (13), 375-392. https://orcid. org/0000-0001-6722-4787

País Andrade, M. (2011). Cultura, Juventud, Identidad: una mirada socioantropológica del Programa Cultural en Barrios. - 1a ed. - Buenos Aires, Argentina: Estudios Sociológicos Editora.

País Andrade, M. y Molina Roldán, A. (Compiladoras). (2013) Cultura y desarrollo en América latina. Actores, estrategias, formación y prácticas. Buenos Aires, Argentina: Ediciones Cooperativas.

País Andrade, M. (2016). Identidades culturales en y desde las fronteras. Un enfoque de género a la(s) políticas y a la(s) práctica(s) culturales juveniles. Buenos Aires, Argentina: Antropofagia.

País Andrade, M. (Compiladora). (2018). Perspectiva de géneros. experiencias interdisciplinarias de intervención/investigación. Buenos Aires, Argentina: Fundación CICUUS.

País Andrade, M. e Igarzábal, B. (editoras). (2021). De la cultura al feminismo. TOMO I y II Colección Culturas Políticas. Serie Cultura y Género(s). Buenos Aires: RCG ediciones.

Peters, T. (2019). “Oferta y consumo cultural. Los desafíos de un concepto sospechoso”. En R. Chavarría et al., (2019), Conceptos Clave de la Gestión Cultural. Enfoques desde Latinoamérica (pp.271-292). Volumen I. Ariadna Ediciones, .

Quijano, A. (2007). “Colonialidad del poder y clasificación social”. En S. Castro-Gómez \& R. Grosfoguel (Eds.), El giro decolonial Reflexiones para una diversidad epistémica más allá del capitalismo global (pp.93-126). Siglo del Hombre Editores.

Rebello, D. (2010.) Programa cultura viva: novos deshenhos de políticas culturais na contemporaneidade. Universidade Federal Do Rio de Janeiro.

Reygadas, L. (2007). “La desigualdad después del (multi)culturalismo”. En A. Giglia, C. Garma \& A., de Teresa (Comps.). ¿Adónde va la antropología? (pp. 341-364). UAM-Iztapalapa.

Rubin, G. (1989). “Notas para una teoría radical de la sexualidad”. en C. Vance (Comp.), Placer y peligro (pp.113-190). Revolución.

Segato, R. (2015). “Introducción: Colonialidad del poder y antropología por demanda”. En R. Segato, La crítica de la colonialidad en ocho ensayos. Y una antropología por demanda (pp.11-34), Prometeo.

Scott, J. W. ([1988]1999). El Género: Una categoría útil para el análisis histórico. Fondo de Cultura Económica. Universidad Autónoma de la Ciudad de México.

Shore, C. (2010). La antropología y el estudio de la política pública: reflexiones sobre la "formulación" de las políticas. Antípoda, (10), 21-49.

Shore, C. \& Wright, S. (1997). “Introduction: Policy. A new field of anthropology”. En Anthropology of Policy: Critical Perspectives on Governance and Power. Routledge, 3-39. 
SInCA. (2018). "Mujeres en la cultura. Notas para el análisis del acceso y la participación cultural en el consumo y el mercado de trabajo". Ministerio de Cultura, Argentina. https:// www.sinca.gob.ar/VerDocumento.aspx? IdCategoria=1

Valencia, S. (2018). El transfeminismo no es un generismo. Pléyade, (22), 27-43. https://dx.doi. org/10.4067/S0719-36962018000200027

Vich, V. (2014). Desculturalizar la cultura. La gestión cultural como forma de acción política. Siglo XXI Editores.

Vich, V. (2018). “¿Qué es un gestor cultural? (En defensa y en contra de la cultura)”. En C. Yáñez (Ed.), Praxis de la gestión cultural (pp.47-54). Universidad Nacional de Colombia.

Viveros, M. (2016). La interseccionalidad: una aproximación situada a la dominación. Debate Feminista, 52, 1-17.

Wright, S. (2004). “La politización de la cultura”. En M. Boivin, A. Rosato \& V. Arribas, Constructores de otredad (pp.128-141). Antropofagia.

Wortman, A. (1996). Repensando las políticas culturales de la transición. Sociedad, 9, 63-84.

Yáñez, C. (Ed.). (2014). Emergencias de la Gestión Cultural en América Latina. Universidad Nacional de Colombia (Sede Manizales), Facultad de Administración, Departamento de Ciencias Humanas.

Yúdice, G. (2002). El recurso de la cultura. Usos de la cultura en la era global. Barcelona: Gedisa.

- Sobre la autora:

Marcela País-Andrade es Licenciada y Profesora en Sociología. Doctora de la Universidad de Buenos Aires (Antropología) y Posdoctora en Ciencias Sociales por la misma Universidad. Investigadora independiente del CONICET en el ICA/FFyL-UBA donde desarrolla diversas investigaciones/intervenciones (trans)feministas en el campo cultural.

\section{- ¿Cómo citar?}

País-Andrade, M. (2021). Tensiones de génerols) y desafíos (trans)feministas: Políticas culturales y gestión pública. Comunicación y Medios, (44), 70-81. https://doi.org/10.5354/07191529.2021 .60956 